\title{
THE MODULARITY OF THE LATTICE OF VARIETIES OF COMPLETELY REGULAR SEMIGROUPS AND RELATED REPRESENTATIONS
}

\author{
by MARIO PETRICH and NORMAN R. REILLY
}

(Received 10 November, 1988)

\begin{abstract}
1. Introduction. A semigroup endowed with a unary operation satisfying the identities

$$
x=x x^{-1} x, \quad x^{-1}=x^{-1} x, \quad\left(x^{-1}\right)^{-1}=x
$$
\end{abstract}

is a completely regular semigroup. In several recent papers devoted to the study of the lattice $\mathscr{L}(\mathscr{C} \mathscr{R})$ of subvarieties of the variety $\mathscr{C} \mathscr{R}$ of completely regular semigroups, various results have been obtained which decompose special intervals in $\mathscr{L}(\mathscr{C} \mathscr{R})$ into either direct products or subdirect products. Petrich [14], Hall and Jones [6] and Rasin [20] have shown that certain intervals of the form $[\mathscr{T}, \mathscr{U} \vee \mathscr{V}]$, where $\mathscr{T}$ is the trivial variety and $\mathscr{V}, \mathscr{V} \in \mathscr{L}(\mathscr{C} \mathscr{R})$ are subdirect products of $[\mathscr{T}, \mathscr{U}]$ and $[\mathscr{T}, \mathscr{V}]$. Pastijn and Trotter [13] show that certain intervals of the form [ $\mathcal{V} \cap \mathscr{V}, \mathcal{U} \vee \mathscr{V}$ ] are direct products of the intervals $[\mathcal{U} \cap \mathcal{V}, \mathcal{U}]$ and $[\mathcal{U} \cap \mathcal{V}, \mathscr{V}$ ]. The main objective of this paper is to develop an appropriate lattice theoretic framework for these representations.

A key observation is the fact that $\mathscr{L}(\mathscr{C} \mathscr{R})$ is modular. This was proved by Pastijn [11] using Polák's Theorem [18]. Since the derivation of Polák's Theorem is quite ardous, we provide an alternative proof of this fact which requires only fairly elementary techniques together with just one of the concepts used in Polák's Theorem but nothing like its full strength. In doing so we establish results of independent interest concerning commuting fully invariant congruences on the free unary semigroup.

The necessary background is introduced in Section 2. In the main result of Section 3, it is established that the interval of congruences between the smallest completely regular congruence and the smallest semilattice congruence on the free unary semigroup is a lattice of commuting congruences.

In Section 4 , taking advantage of the fact that the mapping $\mathscr{V} \rightarrow(\mathscr{V} \cap \mathscr{S}, \mathscr{V} \vee \mathscr{S})$ is an isomorphism of the lattice $\mathscr{L}(\mathscr{C} \mathscr{R})$ onto a subdirect product of the lattice $\mathscr{L}(\mathscr{P})$ of subvarieties of the variety $\mathscr{S}$ of semilattices and the interval $[\mathscr{P}, \mathscr{C} \mathscr{R}]$, it is shown that $\mathscr{L}(\mathscr{C} \mathscr{R})$ is modular.

Section 5 is devoted to the study of the mapping $x \rightarrow(a \wedge x, b \wedge x)(x \in L)$ where $a$ and $b$ are fixed elements of a lattice $L$, and the conditions under which this mapping will be a faithful representation of the ideal generated by $a \vee b$ as a subdirect product of the ideals generated by $a$ and $b$, respectively. These results are then applied to obtain descriptions of the lattices of subvarieties of orthodox cryptogroups and locally orthodox cryptogroups from [14] and [6], [20], respectively.

The main result of Section 6 establishes that if $\kappa$ and $\tau$ are disjoint congruences on a lattice $L$ and the elements $a, b \in L$ are such that $a \kappa a \wedge b \tau b,^{\prime}$ then the mapping $x \rightarrow(x \wedge a, x \wedge b)$ is an isomorphism of $[a \wedge b, a \vee b]$ onto $[a \wedge b, a] \times[a \wedge b, b]$. Various equivalent conditions on $a$ and $b$ are considered. In particular, if $\kappa$ and $\tau$ are complete congruences and $a^{\kappa}, a^{\tau}$ are the maximum elements of $a \kappa$ and $a \tau$, respectively, then $a^{\kappa}$ and $a^{\mathfrak{\tau}}$ satisfy these conditions.

Glasgow Math. J. 32 (1990) 137-152. 
In Section 7 , the results of Section 6 are applied to $\mathscr{L}(\mathscr{C} \mathscr{R})$ by taking advantage of the kernel and trace relations $K, T, T_{l}$ and $T_{r}$. In this way decompositions of the intervals

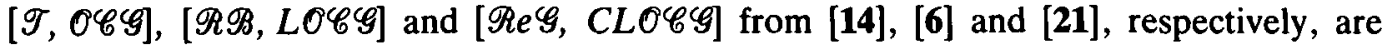
provided with simpler and more systematic derivations.

ACKNOWLEDGEMENT. The authors wish to acknowledge that the question of commuting congruences on the free unary semigroup was not considered in the first verison of this paper. However, this aspect was raised by F. Pastijn in conversation with the second author at the International Conference on Algebra in Lisbon, June 1988. As a result, Section 3 was revised, using the same techniques. The referee pointed out that a similar analysis to that in Section 3 has been performed independently by F. Pastijn and an analysis similar to that in Sections 5,6 and 7 has been performed independently by P. Trotter.

2. Preliminaries. We refer the reader to [8] for basic information, notation and terminology for semigroups. For any semigroup $S$, let $E(S)$ denote the set of idempotents of $S$ and let $\mathscr{C}(S)$ denote the lattice of congruences on $S$. Throughout the following, let $X$ denote a fixed countably infinite set and $U$ denote the free unary semigroup on $X$ (for details, see [3]). The variety $\mathscr{C} \mathscr{R}$ of completely regular semigroups is the subvariety of the variety $\mathcal{U}$ of unary semigroups defined by the identities

$$
x=x x^{-1} x, \quad x x^{-1}=x^{-1} x, \quad x=\left((x)^{-1}\right)^{-1} .
$$

We denote the usual antiisomorphism between the lattice of fully invariant congruences on $U$ and the lattice of unary varieties by $\pi$. Let $\zeta$ be the fully invariant congruence on $U$ corresponding to $\mathscr{C} \mathscr{R}$ under $\pi$. We identify the free completely regular semigroup $F=F \mathscr{C} \mathscr{R}$ on $X$ with $U / \zeta$. The following varieties will be important.

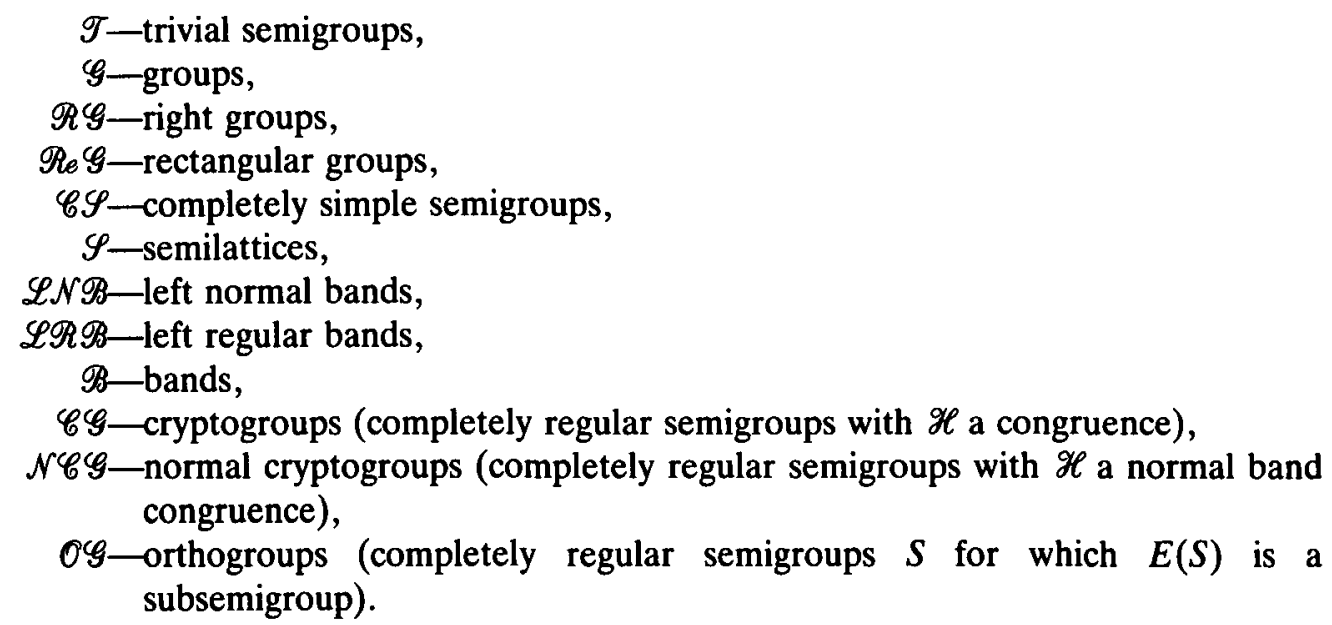

For any variety $\mathscr{V}$, we shall write $\mathscr{L}(\mathscr{V})$ for the lattice of subvarieties of $\mathscr{V}$.

In general, we will write $\zeta_{V}$ for the fully invariant congruence on $U$ corresponding to 
the subvariety $\mathscr{V}$ of $\mathscr{C} \mathscr{R}$. Let

$u^{0}=u u^{-1}$, for any $u \in U$,

$c(u)=$ the set of variables from $X$ appearing in $u \in U$,

$\#(u)=$ the cardinality of $c(u)$,

$h(u)=$ the first element of $X$ to appear in $u$ when read from left to right,

$t(u)=$ the last element of $X$ to appear in $u$ when read from left to right,

$\Delta=\left\{\zeta_{V} \mid \mathscr{V} \in \mathscr{L}(\mathscr{C} \mathscr{R})\right\}$,

$\epsilon=$ identity relation,

$Z^{+}=$the free semigroup on the (non-empty) set $Z$,

$A \backslash B=$ the set of elements in $A$ but not in $B$,

$\varnothing=$ the empty set,

$\circ=$ the relational product.

Let $u \in U$ and $v$ be the largest initial segment of $u$ when viewed as an element in $\left(X \cup\left\{(,)^{-1}\right\}\right)^{+}$containing all but one of the elements of $c(u)$. Then $s(u)$ is the word obtained from $v$ by deleting all unmatched brackets (.

We will be concerned mainly with $\Delta$ and, for any $\rho, \sigma \in \Delta$ with $\rho \subseteq \sigma$ will write

$$
[\rho, \sigma]=\{\tau \in \Delta \mid \rho \subseteq \tau \subseteq \sigma\} .
$$

For any $\mathscr{V} \in \mathscr{L}(\mathscr{C R})$ we define the Mal'cev product of $\mathscr{R} \mathscr{G}$ and $\mathscr{V}$ by

$\mathscr{R} \mathscr{G} \circ \mathscr{V}=\{S \in \mathscr{C} \mathscr{R} \mid$ there exists a congruence $\rho$ on $S$ with $e \rho \in \mathscr{R} \mathscr{G}$, for all $e \in E(S)$, and $S / \rho \in \mathscr{V}\}$.

LEMMA 2.3. Let $\mathscr{V} \in \mathscr{L}(\mathscr{C} \mathscr{R})$.

(i) $\mathscr{R} \mathscr{G} \circ \mathscr{V} \in \mathscr{L}(\mathscr{C} R)$.

(ii) $S \in \mathscr{R} \mathscr{G} \circ \mathscr{V}$ if and only if there exists $\rho \in \mathscr{C}(S)$ with $\rho \subseteq \mathscr{R}$ and $S / \rho \in \mathscr{V}$.

Proof. (i) See (Jones [10], Theorem 5.1).

(ii) This follows easily from the definition.

Two particular Mal'cev products are important for the discussion below. For any property or class $\mathscr{P}$, we will say that $S \in \mathscr{C} \mathscr{R}$ is locally $\mathscr{P}$ if $e S e$ has the property $\mathscr{P}$ or is in the class $\mathscr{P}$ for all $e \in E(S)$. Then we define

$\mathscr{R} \mathscr{R} O \mathscr{G}=\mathscr{R} \mathscr{G} \circ \mathscr{S}$ - the variety of right regular orthogroups,

$L \mathscr{R} \mathscr{R} O \mathscr{G}=\mathscr{R} \mathscr{G} \circ \mathscr{L N} \mathscr{B}$ - the variety of locally right regular orthogroups.

In the remainder of this section we develop tests for $\mathscr{R}$-equivalence in $U / \rho$ $\left(\rho \in\left[\zeta, \zeta_{\varphi}\right]\right)$.

LemMa 2.4 (i) $\zeta_{\mathscr{S}} / \zeta_{\mathscr{R} \mathscr{R} O \mathscr{G}} \subseteq \mathscr{R}$. (ii) $\zeta_{\mathscr{L N} \mathscr{R}} / \zeta_{L \mathscr{R} \mathscr{R} O \mathscr{G}} \subseteq \mathscr{R}$.

Proof. These observations follow easily from the definitions of $\mathscr{R} \mathscr{R} \mathscr{G}$ and $L \mathscr{R} \mathscr{R} O \mathscr{G}$ as Mal'cev products and Lemma 2.3(ii).

Corollary. 2.5. Let $\rho \in\left[\zeta, \zeta_{s}\right]$ and $u, v \in U$.

(i) If $\zeta_{R R O Q G} \subseteq \rho \subseteq \zeta_{\mathscr{S}}$, then

$$
u \rho \mathscr{R} v \rho \Leftrightarrow u \zeta_{s \rho} v \Leftrightarrow c(u)=c(v) .
$$

(ii) If $\zeta_{L R R O \hookrightarrow S} \subseteq \rho \subseteq \zeta_{\mathscr{L N} B}$, then

$$
u \rho \mathscr{R} v \rho \Leftrightarrow u \zeta_{\mathscr{L N} \mathscr{A}} \mathscr{R} v \zeta_{\mathscr{L N} \mathscr{B}} \Leftrightarrow c(u)=c(v) \text { and } h(u)=h(v) .
$$


Proof. (i) Let $u \rho \mathscr{R} v \rho$. Then clearly $u \zeta_{\mathscr{S}} \mathscr{R} v \zeta_{\mathscr{S}}$. But $\mathscr{R}=\epsilon$ in $\mathscr{T}$. Hence $u \zeta_{\mathscr{S}} v$. Conversely, let $u \zeta_{s} v$. Then $(u \rho) \zeta_{s} / \rho(v \rho)$ so that, by Lemma 2.4(i), we have $u \rho \mathscr{R} v \rho$. The second equivalence is well known.

Part (ii) follows similarly.

LEMma 2.6. (i) $\mathscr{L}(\mathscr{C} \mathscr{R})$ is the disjoint union of the intervals $[\mathscr{T}, \mathscr{C} \mathscr{Y}],[\mathscr{Y}, \mathscr{R} \mathscr{R} O \mathscr{G}]$,

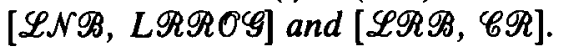

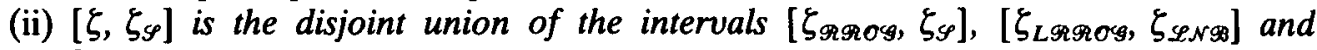
$\left[\zeta, \zeta_{\mathscr{P Q R} B}\right]$.

Proof. (i) This can be found in (Pastijn [11], Lemma 5).

(ii) This follows from (i) via the antiisomorphism $\pi$.

For any $\rho \in\left[\zeta, \zeta_{\mathscr{L} \mathscr{R} B}\right]$, let $\rho^{s}$ denote the relation defined on $U$ by

$$
u \rho^{s} v \Leftrightarrow \text { there exist } w, t \in U \text { with } w \rho t, u=s(w) \text { and } v=s(t) .
$$

We also adopt the convention that $\varnothing \rho^{s} \varnothing$.

LeMma 2.7. Let $\rho \in\left[\zeta, \zeta_{\mathscr{P S R} B}\right]$.

(i) $\rho^{s}$ is a fully invariant congruence.

(ii) The mapping $\rho \rightarrow \rho^{s}$ is a complete endomorphism of $\left[\zeta, \zeta_{\mathscr{L R} \mathscr{B}}\right]$.

(iii) Let $u, v \in U$. Then

$$
u \rho \mathscr{R} v \rho \Leftrightarrow c(u)=c(v) \text { and } s(u) \rho^{s} s(v) .
$$

Proof. (i) See (Polák [17], Theorem 3(4)).

(ii) See (Polák [18], Theorem 1.6(1)).

(iii) This is established in the proof of (Polák [17], Theorem 3(1)).

3. Commutativity in $[\zeta, \omega]$. Recall that congruences $\lambda$ and $\rho$ on an algebra are said to commute if $\lambda \circ \rho=\rho \circ \lambda$. In this section, we consider which congruences $\lambda, \rho$ in $[\zeta, \omega]$ commute. The main result is the following.

TheOREM 3.1. $\left[\zeta_{\mathscr{C} S}, \omega\right]$ and $\left[\zeta, \zeta_{\mathscr{S}}\right]$ are lattices of commuting congruences.

Proof. That $\left[\zeta_{\mathscr{C S}}, \omega\right]$ is a lattice of commuting congruences is a straightforward consequence of the description in (Rasin [19]) of the lattice of fully invariant congruences on the free completely simple semigroup $F_{\mathscr{C} R} / \zeta_{\mathscr{C S} \text {. }}$. So we consider the interval $\left[\zeta, \zeta_{\mathscr{S}}\right]$.

Let $\lambda, \rho \in\left[\zeta, \zeta_{\mathscr{S}}\right]$. Then any $\lambda \circ \rho$-related or $\rho \circ \lambda$-related elements must have the same content. So, for $n \geq 1$, let

$$
K_{n}=\{u \in U: \#(u)=n\} .
$$

We will prove, by induction on $n$ that

$$
\left.\lambda \circ \rho\right|_{K_{n}}=\left.\rho \circ \lambda\right|_{K_{n}} .
$$

First, let $u, v, w \in K_{1}$ be such that

$$
u \lambda v \rho w .
$$

Since $\lambda, \rho \subseteq \zeta_{\varphi}$, it follows that $c(u)=c(w)=\{x\}$, say, so that $u \zeta, v \zeta, w \zeta \in H_{x \zeta}$ and 
we have

$$
(u \zeta) \lambda / \zeta(v \zeta) \rho / \zeta(w \zeta)
$$

But $H_{x \zeta}$ is a group and so the restrictions of $\lambda / \zeta$ and $\rho / \zeta$ to $H_{x \zeta}$ commute. Hence there exists an element $v^{*} \in K_{1}$ such that

$$
(u \zeta) \rho / \zeta\left(v^{*}\right) \zeta \lambda / \zeta(w \zeta)
$$

whence $u \rho v^{*} \lambda w$ so that $u \rho \circ \lambda w$. Thus $\lambda \circ \rho \subseteq \rho \circ \lambda$ on $K_{1}$ and the claim holds for $n=1$ by symmetry.

Now suppose that (1) holds for $n$ and consider the restrictions of $\lambda \circ \rho$ and $\rho \circ \lambda$ to $K_{n+1}$. Let $u, v, w \in K_{n+1}$ be such that (2) holds. Our first goal is to establish the following claim:

$$
\text { there exists } b \in U \text { with } u \rho \mathscr{R} b \rho \text { and } b \lambda \mathscr{R} w \lambda \text {. }
$$

We consider five cases, all but one of which are straightforward.

Case 1. $\zeta_{\mathscr{R} R O S} \subseteq \lambda \subseteq \zeta_{\mathscr{S}}$. In this case $c(u)=c(w)$ and, by Corollary $2.5(\mathrm{i})$, we have $u \rho \mathscr{R} u \rho$ and $u \lambda \mathscr{R} w \lambda$.

Case 2. $\zeta_{\Re \Re O g} \subseteq \rho \subseteq \zeta_{\mathscr{S}}$. In this case $c(u)=c(w)$ and, by Corollary $2.5(\mathrm{i})$, we have $u \rho \mathscr{R} w \rho$ and $w \lambda \mathscr{R} w \lambda$.

Case 3. $\zeta_{L \mathscr{R} \text { ROG }} \subseteq \lambda \subseteq \zeta_{\mathscr{P N} \mathscr{B}}$ and $\rho \subseteq \zeta_{\mathscr{P N B}}$. Then $h(u)=h(v)=h(w)$ and $c(u)=$ $c(w)$. Hence, by Corollary $2.5(\mathrm{ii}), u \rho \mathscr{R} u \rho$ and $u \lambda \mathscr{R} w \lambda$.

Case 4. $\zeta_{L R R O S} \subseteq \rho \subseteq \zeta_{\mathscr{S N R}}$ and $\lambda \subseteq \zeta_{\mathscr{L N O B}}$. Then $h(u)=h(v)=h(w)$ and $c(u)=$ $c(v)$. Hence, by Corollary $2.5(\mathrm{ii}), u \rho \mathscr{R} w \rho$ and $w \lambda \mathscr{R} w \lambda$.

Case 5. $\lambda, \rho \subseteq \zeta_{\mathscr{L} \mathscr{R})}$. From (2), we have that

$$
s(u) \lambda^{s} s(v) \rho^{s} s(w) .
$$

From Lemma 2.7(ii), it follows that $\lambda^{s}, \rho^{s} \in\left[\zeta, \zeta_{\mathscr{S} 9 R}\right]$ and therefore that $c(s(u))=$ $c(s(v))=c(s(w))$. But $\#(s(u))=n$ so that by the induction hypothesis, we can assert that there exists an element $a \in K_{n}$ with

$$
s(u) \rho^{s} a \lambda^{s} s(w) .
$$

Let $z \in X$ be such that $c(p) \backslash c(s(p))=\{z\}$, for $p \in\{u, v, w\}$, and let $b=a z$. Then $a=s(b)$ and $c(b)=c(u)=c(w)$. By Lemma 2.7(iii), $u \rho \mathscr{R} b \rho$ and $b \lambda \mathscr{R} w \lambda$.

By Lemma 2.6(ii), the above cases cover all possibilities and so we can conclude that (3) holds for \#(u)=n+1.

By duality, there exists an element $d \in U$ with $c(d)=c(u)=c(w)$ and

$$
u \rho \mathscr{L} d \rho \text { and } d \lambda \mathscr{L} w \lambda
$$

Hence

$$
\begin{array}{ll}
u^{0} \rho b^{0} u^{0} & \text { by (3) } \\
\rho b^{0} u^{0} d^{0} & \text { by (4). }
\end{array}
$$


Similarly, we have $w^{0} \lambda b^{0} u^{0} d^{0}$. Consequently, with $e=u^{0}, f=v^{0}, g=w^{0}$ and $h=$ $\left(b^{0} u^{0} d^{0}\right)^{0}$, we have $e \rho h \lambda g$.

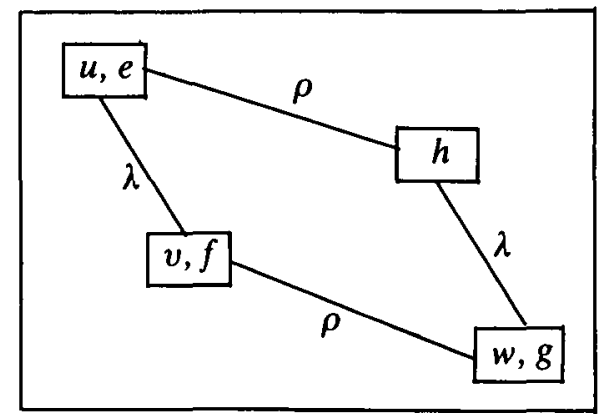

Therefore

Now the elements

$$
\begin{gathered}
u \zeta(e f)^{0} u e \rho(h f)^{0} u h \lambda(h f)^{0} v h, \\
w \zeta(g f)^{0} w g \lambda(h f)^{0} w h \rho(h f)^{0} v h .
\end{gathered}
$$

$$
p=(h f)^{0} u h \zeta, \quad q=(h f)^{0} v h \zeta \quad \text { and } \quad r=(h f)^{0} w h \zeta
$$

all lie in the $\mathscr{H}$-class $H_{h \zeta}$ of $F \mathscr{C} \mathscr{R}$ and, by (5) and (6),

$$
p \lambda / \zeta q \rho / \zeta r \text {. }
$$

Since congruences on a group commute, the restrictions of $\lambda / \zeta$ and $\rho / \zeta$ to $H_{h \zeta}$ commute so that there must be an element $z \in U$, with $s=z \zeta \in H_{h \zeta}$ such that

$$
p \rho / \zeta s \lambda / \zeta r
$$

From (5), (6), (7) and (8), we have

$$
u \rho(h f)^{0} u h \rho z \text { and } w \lambda(h f)^{0} w h \lambda z
$$

so that $u \rho \circ \lambda w$. Thus $\lambda \circ \rho \subseteq \rho \circ \lambda$ on $K_{n+1}$ and, by symmetry, we must have $\lambda \circ \rho=\rho \circ \lambda$ on $K_{n+1}$. By induction, the proof of the theorem is now complete.

The following example illustrates that the result in Theorem 3.1 cannot be extended to cover the whole interval $[\zeta, \omega]$.

EXAMPLE 3.2. The congruences $\zeta_{\mathscr{C S}}$ and $\zeta_{\mathcal{S N} \mathscr{B}}$ do not commute. Let $x, y, p, q$ be distinct elements of $X$. Then

$$
(x p y)^{0} \zeta_{\lessdot \mathscr{S}}(x q y)^{0} \zeta_{\mathscr{S P N B}}(x y q)^{0} .
$$

Now suppose that there is an element $u \in U$ such that

$$
(x p y)^{0} \zeta_{\mathscr{P N} B S} u \zeta_{\mathscr{C S}}(x y q)^{0} .
$$

Since $\zeta_{\mathscr{S} \mathcal{N} \mathscr{B}} \subseteq \zeta_{\mathscr{S}}$, we must have $t(u) \in\{x, p, y\}$. But, for any elements $a, b \in U$, if $a \zeta_{\mathscr{C S}} b$, then necessarily $t(a)=t(b)$. Hence,

$$
q=t\left((x y q)^{0}\right)=t(u) \in\{x, p, y\}
$$

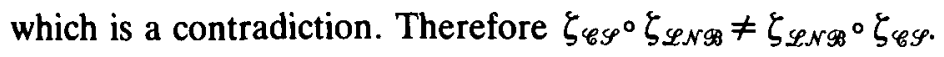


4. Modularity of $\mathscr{L}(\mathscr{C} \mathscr{R})$. In this section we shall show how the modularity of $\mathscr{L}(\mathscr{C} \mathscr{R})$ is a simple consequence of the results of Section 3 . We will require the following two lemmas.

Lemma 4.1 (Birkhoff [1], page 86). Any lattice of commuting congruences on an algebra is modular.

Lemma 4.2 (Hall and Jones [6], Proposition 3.5). The mapping

$$
\chi: \mathscr{V} \rightarrow(\mathscr{V} \cap \mathscr{S}, \mathcal{V} \vee \mathscr{S}) \quad(\mathscr{V} \in \mathscr{L}(\mathscr{C} \mathscr{R}))
$$

is an isomorphism of $\mathscr{L}(\mathscr{C} \mathscr{R})$ onto a subdirect product of $\mathscr{L}(\mathscr{S})$ and $[\mathscr{Y}, \mathscr{C} \mathscr{R}]$.

This prepares the way for an alternative and simpler verification of the following result of Pastijn's [11].

THEOREM 4.3. $\mathscr{L}(\mathscr{C} \mathscr{R})$ is modular.

Proof. By Lemma 4.1 and Theorem 3.1, it follows that the interval $\left[\zeta, \zeta_{\varphi}\right]$ is modular. But $\pi$ induces an antiisomorphism of the interval $\left[\zeta, \zeta_{\mathscr{S}}\right]$ onto the interval $[\mathscr{S}, \mathscr{C} \mathscr{R}]$ so that $[\mathscr{S}, \mathscr{C} \mathscr{R}]$ is also modular. On the other hand, $\mathscr{L}(\mathscr{S})$ is just a two element chain consisting of $\mathscr{S}$ and $\mathscr{T}$. Hence $\mathscr{L}(\mathscr{P})$ is certainly modular. Consequently, by Lemma $4.2, \mathscr{L}(\mathscr{C} \mathscr{R})$ is a subdirect product of two modular lattices and as such is necessarily modular.

Concerning Theorem 4.3 , it is interesting to note similar results concerning other classes of algebras. It is well known that the lattice of varieties of lattices is distributive and that the lattice of varieties of groups is modular. On the other hand, the lattice of varieties of inverse semigroups is not modular ([15], Corollary XII.3.7) while the lattice of varieties of commutative semigroups satisfies no lattice identity ([2], Theorem 2). In the domain of completely regular semigroups, the modularity of $\mathscr{L}(\mathscr{C} \mathscr{S})$ was established in (Rasin [19], Corollary 6) and the modularity of $\mathscr{L}(\mathscr{C} \mathscr{G})$ in (Hall and Jones [6], Theorem 3.1).

In subsequent sections we shall be interested in decompositions of intervals in $\mathscr{L}(\mathscr{C} \mathscr{R})$. It is of some interest to note here that modularity implies, without further assumptions, that certain intervals are isomorphic.

Corollary 4.4. Let $\mathscr{U}, \mathscr{V} \in \mathscr{L}(\mathscr{C} R)$. Then the mappings $\mathscr{W} \rightarrow \mathscr{W} \vee \mathcal{U}$ and $\mathscr{W} \rightarrow$ $\mathscr{W} \cap \mathscr{V}$ are mutually inverse isomorphisms between the intervals $[\mathcal{U} \cap \mathscr{V}, \mathscr{V}]$ and $[\mathcal{u}, \mathcal{u} \vee \mathscr{v}]$.

Proof. Straightforward.

5. Subdirect product representations. One approach used in the study of $\mathscr{L}(\mathscr{C} \mathscr{R})$ has been to describe certain ideals of the form $\mathscr{L}(\mathcal{U} \vee \mathscr{V})$, for suitable $\mathcal{U}, \mathcal{V} \in \mathscr{L}(\mathscr{C} \mathscr{R})$, as particular subdirect products of $\mathscr{L}(\mathscr{U})$ and $\mathscr{L}(\mathscr{V})$. In this section we place this approach in a lattice theoretic setting.

For any lattice $L$ and any $a \in L$, let

$$
[a)=\{x \in L \mid a \leq x\}, \quad(a]=\{x \in L \mid x \leq a\}
$$

and define the mappings

$$
\mu_{a}: x \rightarrow x \wedge a, \quad v_{a}: x \rightarrow x \vee a \quad(x \in L)
$$


The element $a$ is said to be neutral in $L$ if the mapping

$$
\varphi_{a}: x \rightarrow(x \wedge a, x \vee a) \quad(x \in L)
$$

is an isomorphism of $L$ onto a subdirect product of $(a]$ and $[a)$.

In a modular lattice, the test for neutrality can be simplified.

Lemma 5.1 ([5]). Let $L$ be a modular lattice and $a \in L$. Then the following statements are equivalent:

(i) $a$ is neutral;

(ii) $\mu_{a}$ is a homomorphism;

(iii) $v_{a}$ is a homomorphism.

For any elements $a, b$ in a lattice $L$, let

$$
P_{a, b}=\{(x, y) \in(a] \times(b] \mid x \wedge b=y \wedge a\} .
$$

Our first concern regarding $P_{a, b}$ is to determine when it is a sublattice of $(a] \times(b]$. The properties considered in the next observation will be helpful.

LemmA 5.2. Let $L$ be a lattice and $a, b \in L$. The restriction of $\mu_{a \wedge b}$ to $(b]$ is $a$ homomorphism if and only if the restriction of $\mu_{a}$ to $(b]$ is a homomorphism.

Proof. Necessity. For any $x, y \in(b]$,

$$
\begin{aligned}
(x \vee y) \mu_{a} & =(x \vee y) \wedge a=(x \vee y) \wedge(b \wedge a)=(x \vee y) \mu_{a \wedge b} \\
& =x \mu_{a \wedge b} \vee y \mu_{a \wedge b}=(x \wedge a \wedge b) \vee(y \wedge a \wedge b) \\
& =(x \wedge a) \vee(y \wedge a)=x \mu_{a} \vee y \mu_{a}
\end{aligned}
$$

and, since $\mu_{a}$ clearly respects meets, the desired statement holds.

Sufficiency. For any $x, y \in(b]$,

$$
\begin{aligned}
(x \vee y) \mu_{a \wedge b} & =(x \vee y) \wedge a \wedge b=(x \vee y) \wedge a=(x \vee y) \mu_{a} \\
& =x \mu_{a} \vee y \mu_{a}=(x \wedge a) \vee(y \wedge a) \\
& =(x \wedge a \wedge b) \vee(y \wedge a \wedge b)=\left(x \mu_{a \wedge b}\right) \vee\left(y \mu_{a \wedge b}\right)
\end{aligned}
$$

and, since $\mu_{a \wedge b}$ clearly respects meets, the statement holds.

Lemma 5.3. Let $L$ be a lattice and $a, b \in L$. Then $P_{a, b}$ is a subdirect product of $(a]$ and (b] if and only if the restrictions of $\mu_{a}$ to $(b]$ and of $\mu_{b}$ to (a] are both homomorphisms.

Proof. Let $P=P_{a, b}$.

Necessity. Let $x, y \in(b]$. Then $(a \wedge x) \wedge b=a \wedge x=x \wedge a$ so that $(a \wedge x, x) \in P$. Likewise $(a \wedge y, y) \in P$. But $P$ is a sublattice of (a] $\times(b]$ whence $((a \wedge x) \vee(a \wedge y), x \vee y) \in P$. Consequently, by the definition of $P$,

$$
((a \wedge x) \vee(a \wedge y)) \wedge b=(x \vee y) \wedge a
$$

which implies that

$$
a \wedge(x \vee y) \leq(a \wedge x) \vee(a \wedge y)
$$

Since the reverse inequality is clearly valid, it follows that

$$
a \wedge(x \vee y)=(a \wedge x) \vee(a \wedge y)
$$


and, since $\mu_{a}$ clearly respects meets, we see that $\mu_{a}$ is a homomorphism on $(b]$. By symmetry, the direct implication holds.

Sufficiency. Let $(x, y),(u, v) \in P$. Clearly,

while

$$
(x, y) \wedge(u, v)=(x \wedge u, y \wedge v) \in P
$$

$$
(x \vee u) \wedge b=(x \wedge b) \vee(u \wedge b)=(y \wedge a) \vee(v \wedge a)=(y \vee v) \wedge a
$$

so that $(x, y) \vee(u, v)=(x \vee u, y \vee v) \in P$ and $P$ is a sublattice of $(a] \times(b]$. If $x \in(a]$, then $x \wedge b=(x \wedge b) \wedge a$ and $(x, x \wedge b) \in P$. Similarly, for any $y \in(b]$ we have $(y \wedge a, y) \in P$. Thus $P$ is a subdirect product of $(a]$ and $(b]$.

The preceding lemma warns us that, in general, $P_{a, b}$ need not be a sublattice despite the simple formulation. This implies, in particular, that in the representation theorem to follow, it is not sufficient to check that the mappings $\varphi$ and $\psi$ are mutually inverse order isomorphisms.

THEOREM 5.4. Let $L$ be a modular lattice and $a, b \in L$ be neutral in the sublattice $(a \vee b]$. Then $P_{a, b}$ is a subdirect product of ( $\left.a\right]$ and (b] and the mappings

$$
\varphi: z \rightarrow(z \wedge a, z \wedge b), \quad \psi:(x, y) \rightarrow x \vee y
$$

are mutually inverse isomorphisms between $(a \vee b]$ and $P_{a, b}$.

Proof. By Lemma 5.3, $P=P_{a, b}$ is a sublattice of $(a] \times(b]$. Since $a$ and $b$ are neutral in $(a \vee b)$, it is clear that $\varphi$ is a homomorphism of $(a \vee b]$ into $P$ while $\psi$ clearly maps $P$ into $(a \vee b]$. Let $z \in(a \vee b]$. Then

$$
\begin{aligned}
& (a \vee(z \wedge b)) \wedge b=(a \wedge b) \vee(z \wedge b)=(a \vee z) \wedge b, \\
& (a \vee(z \wedge b)) \vee b=a \vee b=(a \vee b) \vee z=(a \vee z) \vee b
\end{aligned}
$$

so that, by the modularity of $L, a \vee(z \wedge b)=a \vee z$. Hence

$$
\begin{aligned}
& ((z \wedge a) \vee(z \wedge b)) \wedge a=(z \wedge a) \vee(z \wedge a \wedge b)=z \wedge a, \\
& ((z \wedge a) \vee(z \wedge b)) \vee a=a \vee(z \wedge b)=a \vee z=z \vee a .
\end{aligned}
$$

By modularity, it follows that $z \varphi \psi=(z \wedge a) \vee(z \wedge b)=z$ and $\varphi \psi$ is the identity mapping on $(a \wedge b]$.

Now let $(x, y) \in P$. Then

$$
(x \vee y) \wedge a=(x \wedge a) \vee(y \wedge a)=x \vee(x \wedge b)=x .
$$

Similarly, $(x \vee y) \wedge b=y$ whence

$$
(x, y) \psi \varphi=((x \vee y) \wedge a, \quad(x \vee y) \wedge b)=(x, y)
$$

so that $\psi \varphi$ is the identity mapping on $P$ and therefore, since both $\varphi$ and $\psi$ are order preserving, $\varphi$ and $\psi$ are mutually inverse isomorphisms.

We now apply these lattice theoretical considerations to $\mathscr{L}(\mathscr{C} \mathscr{R})$. We shall want to refer to some additional varieties:

$O \mathscr{C} G$-orthocryptogroups (orthodox cryptic completely regular semigroups), $e \in E(S))$,

$L O \mathscr{C} \mathscr{G}$-locally orthocryptogroups (all $S \in \mathscr{C} R$ such that $e S e \in \mathscr{O} \mathscr{G}$, for all

$C L O \mathscr{C} G-\{S \in \mathscr{C} \mathscr{R} \mid$ the subsemigroup of $S$ generated by $E(S)$ lies in $L \mathscr{O} \mathscr{C} G\}$.

These varieties appear in some interesting relationships. 
Lemma 5.5. (i) $\mathscr{B} \vee \mathscr{G}=O \mathscr{O} \mathscr{G}$. (ii) $\mathscr{B} \vee \mathscr{C} \mathscr{S}=L O \mathscr{C} \mathscr{G}$. (iii) $\mathscr{O} \mathscr{G} \vee \mathscr{C} \mathscr{S}=C L O \mathscr{C} \mathscr{G}$.

Proof. (i) See (Petrich [14], Lemma 1).

(ii) See (Hall and Jones [6], Corollary 5.4).

(iii) See (Hall and Jones [6], Theorem 5.3 and Reilly [20], Proposition 5.3).

LemMa 5.6. (i) The varieties $\mathscr{G}$ and $\mathscr{C} \mathscr{S}$ are neutral in $\mathscr{L}(\mathscr{C} R)$.

(ii) The variety $\mathscr{B}$ is neutral in $\mathscr{L}(\mathscr{C} \mathscr{G})$.

Proof. It is proved in (Jones [9], Theorems 3.1 and 3.3) that $\mu_{\mathscr{G}}$ and $\mu_{\mathscr{C} S}$ are homomorphisms on $\mathscr{L}(\mathscr{C} \mathscr{R})$ and it follows easily from (Hall and Jones [6], Proposition 3.4) that the restriction to $\mathscr{L}(\mathscr{C} \mathscr{G})$ of $\mu_{\mathscr{B}}$ is also a homomorphism. The claims then follow from Theorem 4.3 and Lemma 5.1.

Corollary 5.7. (i) (Petrich [14], Theorem) The mappings

$$
\mathscr{V} \rightarrow(\mathscr{V} \cap \mathscr{B}, \mathscr{V} \cap \mathscr{G}), \quad(\mathcal{U}, \mathscr{W}) \rightarrow \mathscr{U} \vee \mathscr{W}
$$

are mutually inverse isomorphisms between $\mathscr{L}(\mathcal{O} \mathscr{G})$ and $\mathscr{L}(\mathscr{B}) \times \mathscr{L}(\mathscr{G})$.

(ii) (Hall and Jones [6], Corollary 5.5, Rasin [20], Proposition 1) The mappings

$$
\mathscr{V} \rightarrow(\mathscr{V} \cap \mathscr{B}, \mathscr{V} \cap \mathscr{C} \mathscr{S}), \quad(\mathcal{U}, \mathscr{W}) \rightarrow \mathscr{U} \vee \mathscr{W}
$$

are mutually inverse isomorphisms between $\mathscr{L}(L O \mathscr{C} \mathscr{G})$ and $P_{\mathscr{B}, \mathscr{C} 9}$.

Proof. (i) By Lemma 5.6 and Theorem 5.4, the mappings in (i) are mutually inverse isomorphisms between $\mathscr{L}(\mathscr{B} \vee \mathscr{G})$ and $P_{\mathscr{B}, \mathscr{G}}$. But, by Lemma $5.5, \mathscr{B} \vee \mathscr{G}=\mathscr{O} \mathscr{C} \mathscr{G}$ and, since $\mathscr{B} \cap \mathscr{G}=\mathscr{T}$, we have $P_{\mathscr{B}, \mathscr{G}}=\mathscr{L}(\mathscr{B}) \times \mathscr{L}(\mathscr{G})$.

(ii) By Lemma 5.6 and Theorem 5.4, the mappings in (ii) are mutually inverse isomorphisms between $\mathscr{L}(\mathscr{B} \vee \mathscr{C} \mathscr{S})$ and $P_{\mathscr{B}, \mathscr{G}}$. In addition, by Lemma $5.5, \mathscr{B} \vee \mathscr{C} \mathscr{S}=$ LOCG.

A further result of the genre being considered in this section appears in ([21], Theorem 4.9) where it is shown that $\mathscr{L}(\mathcal{O G} \vee \mathscr{C} \mathscr{S})$ is isomorphic to $P_{\text {OS, }} \mathscr{C S .}$ A consequence of this result is that $\mathscr{O} \mathscr{G}$ is neutral in $\mathscr{L}(\mathscr{O} \mathscr{G} \vee \mathscr{C} \mathscr{S})$. However, no direct simple verification of the neutrality of $\mathscr{O} \mathscr{G}$ in $\mathscr{L}(\mathscr{O} \mathscr{G} \vee \mathscr{C} \mathscr{S})$ is available so that the approach given here does not lead, as yet, to any economy of effort in the derivation of the result.

6. Representing intervals in lattices. In this section we will study certain circumstances under which an interval of the form $[a \wedge b, a \vee b]$ in a lattice may be isomorphic to the product $[a \wedge b, a] \times[a \wedge b, b]$ with a view to applying this to the lattice $\mathscr{L}(\mathscr{C} \mathscr{R})$. The relationships explored in the first lemma are central to these deliberations.

For any complete congruence $\lambda$ on a complete lattice $L$ and any $a \in L$, the class $a \lambda$ is an interval. We define $a_{\lambda}$ and $a^{\lambda}$ by $a \lambda=\left[a_{\lambda}, a^{\lambda}\right]$.

Lemma 6.1. Let $K$ and $\tau$ be congruences on $a$ lattice $L$ and $a, b \in L$. The following statements are equivalent.

$\begin{array}{ll}\text { (i) } a \kappa a \wedge b \tau b . & \text { (ii) } a \tau a \vee b \kappa b .\end{array}$

Suppose further that $L$ is a complete lattice and that $\mathrm{K}$ and $\tau$ are complete congruences. 
Then (i) and (ii) are equivalent to each of the following statements.
(iii) $a_{x} \leq b \leq a^{\tau}$.
(v) $a \leq b^{\kappa}, b \leq a^{\tau}$.
(iv) $a_{\kappa} \leq b, b_{\tau} \leq a$.
(vi) $b_{\tau} \leq a \leq b^{\kappa}$.

Proof. If (i) holds, then

$$
a=a \vee(a \wedge b) \tau a \vee b, \quad b=(a \wedge b) \vee b \kappa a \vee b
$$

which gives (ii). The argument to show that (ii) implies (i) is similar. Now let $L$ be a complete lattice and $\kappa$ and $\tau$ be complete congruences.

If (ii) holds, then

$$
a_{\kappa} \leq(a \vee b)_{\kappa}=b_{\kappa} \leq b \leq a \vee b \leq(a \vee b)^{\tau}=a^{\tau}
$$

which yields (iii). If (iii) holds, then $b_{\tau} \leq\left(a^{\tau}\right)_{\tau}=a_{\tau} \leq a$ and (iv) holds. If (iv) holds, then

$$
a \leq a^{\kappa}=\left(a_{\kappa}\right)^{\kappa} \leq b^{\kappa} \text { and } b \leq b^{\tau}=\left(b_{\tau}\right)^{\tau} \leq a^{\tau}
$$

so that (v) holds. If (v) holds, then $b_{\tau} \leq\left(a^{\tau}\right)_{\tau}=a_{\tau} \leq a$ whence (vi) holds. Finally assume that (vi) holds. Then

$$
a_{\kappa} \leq\left(b^{\kappa}\right)_{\kappa}=b_{\kappa} \leq b .
$$

But we also have $a_{\kappa} \leq a$. Hence $a_{\kappa} \leq a \wedge b \leq a$ which implies that a $k a \wedge b$. From $b_{\tau} \leq a$ we deduce that $b_{\tau} \leq a \wedge b \leq b$ so that $b \tau a \wedge b$ and (i) follows.

If $L, a, b, \kappa$ and $\tau$ satisfy (i) and (ii) in Lemma 6.1, then we will say that $a$ and $b$ are $\kappa \tau$-neighbours.

Congruences $x$ and $\tau$ on a lattice $L$ are said to be disjoint if $k \cap \tau=\epsilon$.

It is important to note that the property of being neighbours is invariant under dualization. For any lattice $L$, its dual $L^{*}$ is the lattice obtained from $L$ by interchanging the operations $\vee$ and $\wedge$.

COROLlaRY 6.2. Let $\kappa, \tau$ be (disjoint) congruences on a lattice L. Then $\kappa, \tau$ are (disjoint) congruences on $L^{*}$. If $a, b \in L$ are $\kappa \tau$-neighbours in $L$, then they are $\tau \kappa$-neighbours in $L^{*}$.

LeMMA 6.3. Let $\mathrm{K}$ and $\tau$ be disjoint complete congruences on a complete lattice $L$ and let $a \in L$. Then

$$
a=a_{\kappa} \vee a_{\tau}=a^{\kappa} \wedge a^{\tau} .
$$

Proof. Since $k$ and $\tau$ are congruences, we have

$$
a_{\kappa} \vee a_{\tau} \kappa a \vee a_{\tau}=a \text { and } a_{\kappa} \vee a_{\tau} \tau a_{\kappa} \vee a=a
$$

so that $a_{\kappa} \vee a_{\tau}(\kappa \cap \tau) a$. But $\kappa$ and $\tau$ are disjoint. Therefore $a=a_{\kappa} \vee a_{\tau}$. The second equality follows by duality.

COROLlaRY 6.4. Let $\mathrm{K}$ and $\tau$ be disjoint complete congruences on a complete lattice $L$ and let $a \in L$. Then $a^{\kappa}, a^{\tau}$ are $\kappa \tau$-neighbours and $a_{k}, a_{\tau}$ are $\tau \kappa$-neighbours.

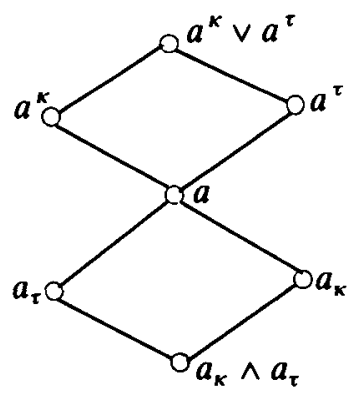


Proof. By Lemma 6.3, we have

$$
a^{\kappa} \wedge a^{\tau}=a \kappa a^{\kappa} \text { and } a^{\kappa} \wedge a^{\tau}=a \tau a^{\tau}
$$

from which we deduce the first claim. The second claim follows similarly using Lemmas 6.3 and 6.1 (ii).

Lemma 6.5. Let $\kappa, \tau$ be disjoint congruences on a lattice $L$ and $a, b \in L$, be $\kappa \tau$-neighbours. Then the mappings

$$
\varphi: z \rightarrow(z \wedge a, z \wedge b), \quad \psi:(x, y) \rightarrow x \vee y
$$

are mutually inverse mappings between $(a \vee b]$ and $P_{a, b}$.

Proof. Clearly $\varphi$ maps $(a \vee b]$ into $P_{a, b}$ and $\psi$ maps $P_{a, b}$ into $(a \vee b]$. Since $a$ and $b$ are $k \tau$-neighbours we have

$$
a \kappa a \wedge b \text { and } a \tau a \vee b \kappa b .
$$

For $(x, y) \in P_{a, b}$, we get

$$
\begin{aligned}
(x \vee y) \wedge a & =(x \vee(y \wedge b)) \wedge a \tau(x \vee(y \wedge a \wedge b)) \wedge(a \vee b) \\
& =x \vee(x \wedge b \wedge b)=x, \\
(x \vee y) \wedge a & =((x \wedge a) \vee y) \wedge a \kappa((x \wedge a \wedge b) \vee y) \wedge a \\
& =((y \wedge a \wedge a) \vee y) \wedge a=y \wedge a=x \wedge b \\
& \kappa x \wedge(a \vee b)=x .
\end{aligned}
$$

Thus $(x \vee y) \wedge a(\kappa \cap \tau) x$ so that $(x \wedge y) \wedge a=x$. Similarly $(x \vee y) \wedge b=y$ whence $\psi \varphi$ is the identity mapping on $P_{a, b}$.

For $z \in(a \vee b]$, we have

$$
\begin{aligned}
(z \wedge a) \vee(z \wedge b) & \tau(z \wedge(a \vee b)) \vee(z \wedge a \wedge b) \\
= & z \vee(z \wedge a \wedge b)=z, \\
(z \wedge a) \vee(z \wedge b) & \kappa(z \wedge a \wedge b) \vee(z \wedge(a \vee b)) \\
= & (z \wedge a \wedge b) \vee z=z .
\end{aligned}
$$

Therefore $(z \wedge a) \vee(z \wedge b)(\kappa \cap \tau) z$ so that $(z \wedge a) \vee(z \wedge b)=z$ and $\varphi \psi$ is the identity on $(a \vee b]$.

We are now ready for the main theorem of this section. One of the striking features of this result is the fact that neither modularity nor neutrality appear in the hypotheses.

THEOREM 6.6. Let $\kappa, \tau$ be disjoint congruences on $a$ lattice $L$ and $a, b \in L$ be $\kappa \tau$-neighbours. Then the mappings

$$
\varphi: z \rightarrow(z \wedge a, z \wedge b), \quad \psi:(x, y) \rightarrow x \vee y
$$

are mutually inverse isomorphisms between $[a \wedge b, a \vee b]$ and $[a \wedge b, a] \times[a \wedge b, b]$.

Proof. Clearly $\varphi$ maps $A=[a \wedge b, a \vee b]$ into $B=[a \wedge b, a] \times[a \wedge b, b]$ and $\psi$ maps $B$ into $A$. Since $(x, y) \in B$ implies that $x \wedge b=a \wedge b=y \wedge a$, it follows that $B \subseteq P_{a, b}$. Hence, by Lemma 6.5, $\varphi$ and $\psi$ are mutually inverse bijections. To complete the proof, it suffices to show that $\varphi$ is a homomorphism since $\psi$, as the inverse mapping, will then automatically be a homomorphism. To show that $\varphi$ is a homomorphism it suffices, by 
symmetry, to show that $\mu_{a}$ is a homomorphism on $A$. So let $x, y \in A$. Then

and

$$
\begin{aligned}
(x \wedge a) \vee(y \wedge a) & \kappa(x \wedge(a \wedge b)) \vee(y \wedge(a \wedge b)) \\
= & a \wedge b \\
= & (x \vee y) \wedge(a \wedge b) \\
& \kappa(x \vee y) \wedge a,
\end{aligned}
$$

$$
\begin{aligned}
(x \wedge a) \vee(y \wedge a) & \tau(x \wedge(a \vee b)) \vee(y \wedge(a \vee b)) \\
= & x \vee y \\
= & (x \vee y) \wedge(a \vee b) \\
& \tau(x \vee y) \wedge a .
\end{aligned}
$$

Since $\kappa \cap \tau=\epsilon$, we conclude from (9) and (10) that

$$
(x \wedge a) \vee(y \wedge a)=(x \vee y) \wedge a .
$$

Thus $\mu_{a}$ respects joins. Clearly $\mu_{a}$ respects meets so that $\mu_{a}$ is a homomorphism on $A$, as required.

7. Local decompositions of $\mathscr{L}(\mathscr{C} \mathscr{R})$. For any equivalence relation $\theta$ on a completely regular semigroup $S$, let $\theta^{\circ}$ denote the largest congruence on $S$ contained in $\theta$. For any congruence $\rho$ on $S$, we define the kernel, trace, left race and right trace of $\rho$ to be

$$
\begin{aligned}
& \operatorname{ker} \rho=\left\{a \in S \mid a \rho a^{0}\right\}, \quad \operatorname{tr} \rho=\left.\rho\right|_{E(S)}, \\
& \operatorname{lt} \rho=\operatorname{tr}(\rho \vee \mathscr{L})^{0}, \quad \operatorname{rtr} \rho=\operatorname{tr}(\rho \vee \mathscr{R})^{0},
\end{aligned}
$$

respectively. Define the relations $K, T, T_{l}$ and $T_{r}$ on $\mathscr{C}(S)$ as follows: for $\lambda, \rho \in \mathscr{C}(S)$,

$$
\begin{array}{ll}
\lambda K \rho \Leftrightarrow \operatorname{ker} \lambda=\operatorname{ker} \rho, & \lambda T \rho \Leftrightarrow \operatorname{tr} \lambda=\operatorname{tr} \rho, \\
\lambda T_{l} \rho \Leftrightarrow \operatorname{ttr} \lambda=\operatorname{ltr} \rho, & \lambda T_{r} \rho \Leftrightarrow \operatorname{rtr} \lambda=\operatorname{rtr} \rho .
\end{array}
$$

In what follows, let $\Gamma$ denote the lattice of fully invariant congruences on $F$, the free completely regular semigroup on $X$. Clearly $\Gamma=\left\{\zeta_{\mathscr{V}} / \zeta \mid \mathscr{V} \in \mathscr{L}(\mathscr{C} \mathscr{R})\right\}$ and we will write $\rho_{\mathscr{V}}=\zeta_{\mathscr{V}} / \zeta$.

Lemma 7.1. (i) The relations $K, T, T_{l}$ and $T_{r}$ are complete congruences on $\Gamma$.

(ii) $K \cap T=\epsilon$.

(iii) $T_{l} \cap T_{r}=T$.

Proof. (i) The claim regarding $K$ was established in (Pastijn [11], Theorem 11), that for $T_{i}$ and $T_{r}$ was established in (Pastijn and Petrich [12], Corollary 4.9) and that for $T$ follows from the preceding observations and part (iii).

For (ii), see (Feigenbaum [4], Theorem 4.1).

For (iii), see (Pastijn and Petrich [12], Corollary 4.8).

TheOREM 7.2. (Pastijn and Trotter [13], Theorems 5.1 and 5.2) Let $\rho \in \Gamma$.

(i) The mappings

$$
\theta \rightarrow\left(\theta \cap \rho^{K}, \theta \cap \rho^{T}\right), \quad(\xi, \eta) \rightarrow \xi \vee \eta
$$

are mutually inverse isomorphisms between $\left[\rho, \rho^{K} \vee \rho^{T}\right]$ and $\left[\rho, \rho^{K}\right] \times\left[\rho, \rho^{T}\right]$. 
(ii) The mappings

$$
\theta \rightarrow\left(\theta \vee \rho_{K}, \theta \vee \rho_{T}\right), \quad(\xi, \eta) \rightarrow \xi \cap \eta
$$

are mutually inverse isomorphisms between $\left[\rho_{K} \cap \rho_{T}, \rho\right]$ and $\left[\rho_{K}, \rho\right] \times\left[\rho_{T}, \rho\right]$.

Proof. (i) From Lemma 7.1, we know that $K$ and $T$ are disjoint complete congruences on $\Gamma$. It follows from Corollary 6.4 that $\rho^{K}$ and $\rho^{T}$ are $K T$-neighbours and the claim follows by Theorem 6.6 .

(ii) This follows from (i) by duality.

TheORem 7.3. Let $\rho \in \Gamma$. Then the mappings

$$
\theta \rightarrow\left(\theta \vee \rho_{T_{l}}, \theta \vee \rho_{T_{r}}\right), \quad(\xi, \eta) \rightarrow \xi \cap \eta
$$

are mutually inverse isomorphisms between $\left[\rho_{T_{i}} \cap \rho_{T_{r}}, \rho\right] \cap \rho K$ and $\left(\left[\rho_{T_{l}}, \rho\right] \cap \rho K\right) \times\left(\left[\rho_{T}, \rho\right] \cap \rho K\right)$.

Proof. By Lemma 7.1(ii) and (iii), the restrictions of $T_{l}$ and $T_{r}$ to $\rho K$ are disjoint complete congruences and so an argument similar to that for Theorem 7.2(ii) will establish the claim.

We now wish to provide some specific illustrations of the preceding discussions but in terms of varieties rather than fully invariant congruences.

By means of the standard antiisomorphism between $\Gamma$ and $\mathscr{L}(\mathscr{C} \mathscr{R})$, the congruences $K$ and $T$ on $\Gamma$ determine disjoint complete congruences, which we will also denote by $K$ and $T$, on $\mathscr{L}(\mathscr{C} \mathscr{R})$ as follows:

$$
\begin{array}{ll}
u K \mathscr{V} \Leftrightarrow \rho_{\mathscr{U}} K \rho_{V} & (\mathcal{U}, \mathcal{V} \in \mathscr{L}(\mathscr{C} \mathscr{R})), \\
u T \mathscr{V} \Leftrightarrow \rho_{u} T \rho_{V} & (U, \mathcal{V} \in \mathscr{L}(\mathscr{C} \mathscr{R})) .
\end{array}
$$

For any $\rho \in \Gamma$, let $[\rho]$ denote the subvariety of $\mathscr{C} \mathscr{R}$ defined by $\rho=\rho_{[\rho]}$. Then, for any $\mathscr{V} \in \mathscr{L}(\mathscr{C} \mathscr{R})$ and writing $\rho=\rho_{\mathscr{V}}$, we have

$$
\mathscr{V}^{K}=\left[\rho_{K}\right] \text { and } \mathscr{V}_{K}=\left[\rho^{K}\right]
$$

Lemma 7.4. (i) $\mathscr{T}^{K}=\mathscr{B}, \quad \mathscr{G}^{K}=\mathscr{R} e^{\mathscr{C}} \mathscr{G}^{K}=\mathscr{O} \mathscr{G}$.

(ii) $\mathscr{T}^{T}=\mathscr{G}, \quad \mathscr{R} \mathscr{B}^{T}=\mathscr{R e} \mathscr{G}^{T}=\mathscr{C} \mathscr{Y}$.

Proof. (i) See (Polák [17], Theorem 2).

(ii) See (Petrich and Reilly [16], Section 9).

Theorem 7.5. (i) (Petrich [14], Theorem) The mappings

$$
\mathscr{V} \rightarrow(\mathscr{V} \cap \mathscr{R}, \mathscr{V} \cap \mathscr{G}), \quad(\mathcal{U}, \mathscr{W}) \rightarrow \mathcal{U} \vee \mathscr{W}
$$

are mutually inverse isomorphisms between $\mathscr{L}(\mathscr{O C G})$ and $\mathscr{L}(\mathscr{B}) \times \mathscr{L}(\mathscr{G})$.

(ii) (Hall and Jones [6], Corollary 5.5 and Rasin [20], Proposition 1) The mappings

$$
\mathscr{V} \rightarrow(\mathscr{V} \cap \mathscr{B}, \mathscr{V} \cap \mathscr{C} \mathscr{S}), \quad(\mathcal{U}, \mathscr{W}) \rightarrow \mathscr{U} \vee \mathcal{W}
$$

are mutually inverse isomorphisms between $[\mathscr{R} \mathscr{B}, \mathscr{O} \mathscr{C} \mathscr{G}]$ and $[\mathscr{R} \mathscr{B}, \mathscr{B}] \times[\mathscr{R} \mathscr{B}, \mathscr{C} \mathscr{S}]$.

(iii) (Reilly [21], Theorem 4.9) The mappings

$$
\mathscr{V} \rightarrow(\mathscr{V} \cap \mathcal{O} \mathscr{G}, \mathscr{V} \cap \mathscr{C} \mathscr{S}) \quad(\mathcal{U}, \mathscr{W}) \rightarrow \mathcal{U} \vee \mathcal{W}
$$

are mutually inverse isomorphisms between $[\mathscr{R e} \mathscr{G}, C L \mathscr{O} \mathscr{G}]$ and $[\mathscr{R e} \mathscr{G}, \mathcal{O} G] \times[\mathscr{R} \mathscr{G}, \mathscr{C} \mathscr{G}]$. 
Proof. (i) This same result was obtained in Corollary 5.7(i) using the subdirect product representation technique of Theorem 5.4. We provide an alternative proof here based on Theorem 6.6 which will serve as a model for the proofs of parts (ii) and (iii). Since $\mathscr{B} \cap \mathscr{G}=\mathscr{T}$ while $\mathscr{T}^{K}=\mathscr{B}$ and $\mathscr{T}^{T}=\mathscr{G}$, by Lemma 7.4 , it follows that $\mathscr{B}$ and $\mathscr{G}$ are $K T$-neighbours. By Lemma 5.5(i), $\mathscr{B} \vee \mathscr{G}=\mathscr{O} \mathscr{C} \mathscr{G}$. The result then follows from Theorem 6.6 .

The proofs of (ii) and (iii) follow the same pattern using the appropriate parts of Lemmas 7.4 and 5.5.

Clearly, if $\mathscr{U}, \mathcal{V} \in \mathscr{L}(\mathscr{C} \mathscr{R})$ are both neutral in $\mathscr{L}(\mathcal{U} \vee \mathscr{V})$, then $\mathcal{U}$ and $\mathscr{V}$ are neutral in $[\mathcal{U} \cap \mathscr{V}, \mathcal{U} \vee \mathscr{V}]$. When $\mathcal{U} \cap \mathcal{V}=\mathscr{T}$, these two concepts coincide and it is natural to wonder if they coincide throughout $\mathscr{L}(\mathscr{C} \mathscr{R})$. To see that this is not the case, let $\mathcal{U}, \mathscr{V}$, $\mathscr{W} \in \mathscr{L}(\mathscr{G})$ be such that $\mathscr{U} \cap(\mathscr{V} \vee \mathscr{W}) \neq(\mathcal{U} \cap \mathscr{V}) \vee(\mathscr{U} \cap \mathcal{W})$ (since $\mathscr{L}(\mathscr{G})$ is not distributive, there must exist such varieties; see [7]). Then we have

$$
\mathcal{U} K \boldsymbol{U}=\mathscr{G} \cap \mathcal{U} \mathscr{G}
$$

so that $\mathscr{U}$ and $\mathscr{G}$ are $K T$-neighbours. However, by the choice of $\mathscr{U}$, we know that $\mu_{\mathscr{U}}$ is not a homomorphism on $\mathscr{L}(\mathscr{G})$ so that $\mathcal{U}$ is not neutral in $\mathscr{L}(\mathscr{G})$. Thus Theorem 6.6 is applicable (in a trivial sort of a way) but Theorem 5.4 is not.

\section{REFERENCES} 1964.

1. G. Birkhoff, Lattice Theory, Amer. Math. Soc. Colloq. Publ. Vol. XXV, Providence,

2. S. Burris and E. Nelson, Embedding the dual of $\Pi_{m}$ in the lattice of equational classes of commutative semigroups, Proc. Amer. Math. Soc. 30 (1971), 37-39. 434-451.

3. A. H. Clifford, The free completely regular semigroup on a set, J. Algebra 59 (1979),

4. R. Feigenbaum, Regular semigroup congruences, Semigroup Forum 17 (1979), 371-377.

5. G. Grätzer, General lattice theory, (Academic Press 1978).

6. T. E. Hall and P. R. Jones, On the lattice of varieties of bands of groups, Pacific J. Math. 91 (1980), 327-337.

7. G. Higman, Representations of general linear groups and varieties of p-groups, Proc. Int. Conf. on the Theory of Groups, Gordon and Breach, New York (1967), 167-173.

8. J. M. Howie, An Introduction to Semigroup Theory, (Academic Press, 1976).

9. P. R. Jones, On the lattice of varieties of completely regular semigroups, J. Austral. Math. Soc. A35 (1983), 227-235.

10. P. R. Jones, Mal'cev products of varieties of completely regular semigroups, J. Austral. Math. Soc. A42 (1987), 227-246.

11. F. Pastijn, The lattice of completely regular semigroup varieties (preprint).

12. F. Pastijn and M. Petrich, Congruences on regular semigroups, Trans. Amer. Math. Soc. 295 (1986), 607-633.

13. F. Pastijn and P. G. Trotter, Lattices of completely regular semigroup varieties, Pacific $J$. Math. 119 (1985), 191-214.

14. M. Petrich, Varieties of orthodox bands of groups, Pacific J. Math. 58 (1975), 209-217.

15. M. Petrich, Inverse Semigroups, (Wiley Interscience, 1984).

16. M. Petrich and N. R. Reilly, Semigroups generated by certain operators on varieties of completely regular semigroups, Pacific J. Math. 132 (1988), 151-175. 97-123.

17. L. Polák, On varieties of completely regular semigroups I, Semigroup Forum 32 (1985),

18. L. Polák, On varieties of completely regular semigroups II, Semigroup Forum 36 (1987), 253-284. 
19. V. V. Rasin, Free completely simple semigroups, Ural. Gos. Univ. Mat. Zap. 11 (1979), 140-151 (Russian).

20. V. V. Rasin, On the varieties of Cliffordian semigroups, Semigroup Forum 23 (1981), 201-220.

21. N. R. Reilly, Varieties of completely regular semigroups, J. Austral. Math. Soc. A38 (1985), 372-393.

Simon Fraser University, BURNABY, B.C.,

Canada. 